\title{
Flux-closure-domain states and demagnetizing energy de- termination in sub-micron size magnetic dots
}

\author{
P.-O. Jubert ${ }^{1}(*)\left(^{* *}\right)$, J.-C. Toussaint ${ }^{1}$, O. Fruchart ${ }^{1}$, C. Meyer ${ }^{1}$ and Y. Sam- \\ $\mathrm{SON}^{2}$ \\ 1 Laboratoire Louis Néel (CNRS, UPR5051) - BP166, 38042 Grenoble Cedex 9, France \\ 2 DRFMC/SP2M/NM (CEA) - 17 rue des Martyrs, F-38054 Grenoble Cedex 9, France
}

PACS. 75.75.+a - Magnetic properties of nanostructures.

PACS. 75.70.Kw - Domain structure (including magnetic bubbles).

\begin{abstract}
We used single-crystalline Fe dots self-assembled under UHV as a model system to discuss micromagnetic properties of sub-micron size magnetic dots and show what properties may or may not be scaled down from macroscopic samples. Landau and diamond states were identified by MFM and reproduced by simulations. These states are surprisingly well reproduced by the the Van den Berg model despite the small dots'size. On the contrary, it is argued theoretically that the usual determination procedure of demagnetizing factors $N_{i}$ 's using initial susceptibility or the over-loop area underestimate the real values of $N_{i}$ 's in sub-micron size dots. This point is confirmed both experimentally and in simulations.
\end{abstract}

The confinement of magnetic domains in finite-size flat elements made of soft magnetic materials has been extensively addressed [1-3]. Up to recently mostly samples of size above some microns had been studied, ignoring the internal structure of domain walls and vortices, that were treated as singularities in [2,3]. Today the improved resolution of magnetic imaging [4,5] and the increased power of computers allow experiments and micromagnetic simulations to overlap in the range $50-1000 \mathrm{~nm}$, while approached analytical models taking into account the internal structure of magnetic non-homogeneities are being developed [6-8]. Therefore, sub-micron size magnetic dots may now be used as model systems for unravelling the microscopic origin of phenomena described up to now phenomenologically in materials, such as nucleation and annihilation events.

The magnetization process of dots depends on both intrinsic (shape, anisotropy) and extrinsic parameters (microstructure, defects). Here we name intrinsic features which are generally part of the design of a system, and extrinsic features which are generally not designed and/nor controllable. Although the influence of extrinsic parameters has been outlined for both annihilation/nucleation events, shape effects and micromagnetic configuration $[9,10]$, the influence of purely intrinsic parameters has been difficult to address experimentally due to defects found in polycrystalline or amorphous dots patterned by lithography. We propose to

(*) pju@zurich.ibm.com

(**) Present address: IBM Research, Zürich Research Laboratory - Säumerstrasse 4, 8803 Rüschlikon, Switzerland.

(C) EDP Sciences 
address these issues using epitaxial self-assembled ingot-shaped Fe dots fabricated and encapsulated under ultra-high vacuum using pulsed laser deposition [11]. These dots (Fig. 11a) display atomically-flat facets [11], bulk lattice parameter and bulk magnetic anisotropy $(<100>$ easy axes), and the inter-dots dipolar interactions are negligible. In this Letter we report some effects of shape and finite size on remanent and in-field magnetization states of such sub-micron size model magnetic dots. We focus on dots approximately $600 \times 300 \times 60 \mathrm{~nm}$. Hysteresis loops were performed at $300 \mathrm{~K}$ over assemblies of dots by Vibrating Sample Magnetometry. Magnetic force microscopy (MFM) was performed at $300 \mathrm{~K}$ using a Nanoscope IIIA (Multimode) from Digital Instruments with a lift height of about $25 \mathrm{~nm}$ and a Si tip coated with $40 \mathrm{~nm}$ of vertically magnetized CoCr. Micromagnetic simulations were performed at $0 \mathrm{~K}$ (no thermal noise) by integrating LLG equations using a custom-developed finite differences code. The sample was divided into $128 \times 64 \times 16$ parallelepipedic cells with lateral and vertical size $h_{x}=h_{y}=4.6875 \mathrm{~nm}$ and $h_{z}=3.75 \mathrm{~nm}$, respectively. The magnetization vector at each node is estimated as a second order interpolation of the vector field between the cell and its nearest neighbors, thus allowing the volume charges to vary linearly. The accuracy of our approach reaches the precision of the model B proposed by Ramstöck et al. [12]. In the reported calculations the dots edges were taken vertical to avoid discretizing artifacts on the side facets and simplify the comparison with flat dots. No significant difference was found for calculations performed with tilted facets. The magnetic anisotropy was that of bulk Fe and the external field was applied $0.1^{\circ}$ off high symmetry directions to avoid numerical artifacts.

Hysteresis loops of assemblies of dots were found to display zero remanence in any direction [11]. Mostly two types of remanent states were observed when imaging an assembly of dots at zero external field with MFM (Fig. 1 b). Simulated MFM contrast assuming a unipolar tip (Fig. 1 1 c) allows us to identify the so-called Landau state (LS, left) and diamond state (DS, right). These two states are well known for flat dots well above micron-size, and are explained by the Van den Berg (VdB) model [2]. Surprisingly the locus of magnetic walls and vortices in the simulated states is very close to the predictions of $\mathrm{VdB}$ down to tiny details like the slight vertical elongation of the two vortices in the DS (Fig. 11d). Indeed the VdB model relies on three hypothesis, none of which is fulfilled in our case: infinitely soft magnetic material, infinitely thin plate, infinitely large sample (the latter being equivalent to zero exchange). Paradoxically other micron-size systems that fulfill all three criteria better than the present dots, like thin micron-size plates of Permalloy, were shown to deviate from VdB predictions [13]. We explain this apparent contradiction the following way. Firstly dipolar energy increases with the dot's height, so that both dipolar and anisotropy energies compress domain walls, reducing characteristic length scales (and removing domain wall tails) to a scale smaller than the dot's lateral size. Secondly, as in our case dipolar energy dominates over anisotropy energy, the locus of the walls and vortices is determined mainly by dipolar energy, and therefore coincides with $\mathrm{VdB}$ predictions.

So far we have ignored the internal structure of walls and vortices. Let us examine crosssections of both LS and DS. Interesting are the top and bottom plane views of the LS that look very different from the mid-height view (Fig. 2a), due to the formation of Néel caps that reduce the amount of magnetic charges at both surfaces. The lateral cross section along the long direction $x$ (Fig. $2 \mathrm{~b}$ ) reveals that the Bloch wall has the same topology as a single vortex: starting from a vortex, one can 'fabricate' a wall by driving apart the top and bottom ends and stretch laterally the mid-height core. A close examination of the middle plane view in (a) also shows that the wall is slightly bent downwards, although equilibrium is reached in the simulation. As is better revealed by Fig. 20 the wall is an asymmetric Bloch wall, that helps reducing volume magnetic charges (arising from Néel caps) at the expense of exchange [1]. MFM resolution is not high enough to confirm that the experimental Bloch walls 
(a)

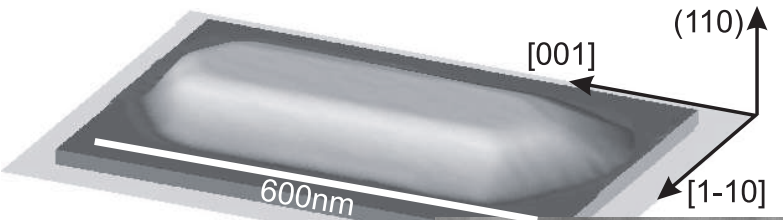

(b)

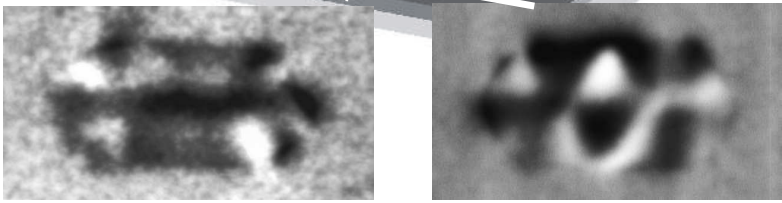

(c)

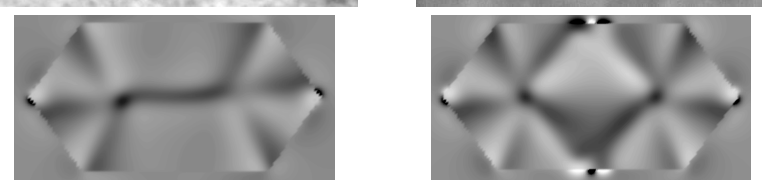

(d)

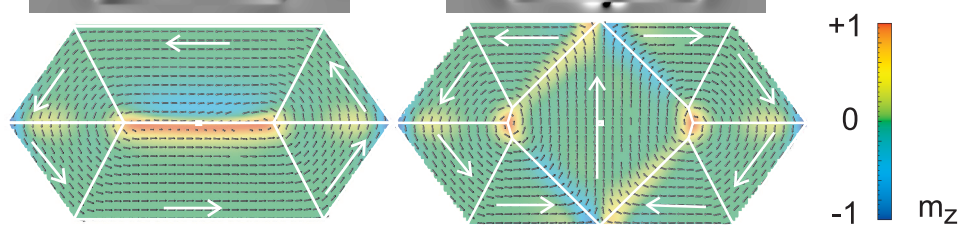

Fig. $1-$ (a) $3 \mathrm{D}$ AFM image of a $600 \times 300 \times 60 \mathrm{~nm}$ Fe dot (true vertical scale). Below are shown (b) experimental MFM images after saturation along [001], (c) simulated $\mathrm{d} H / \mathrm{d} z$ maps over the dot with a lift height of $30 \mathrm{~nm}$, and (d) simulated configurations superimposed here with the Van den Berg construction, for both Landau (left) and diamond (right) states. Note the bipolar contrast of Néel walls and the unipolar contrast of the Bloch wall in (b-c). In (d) the color reveals the perpendicular component of magnetization of the mid-height plane, while white arrows sketch the in-plane magnetization direction.

are asymmetric. The vortices in the DS are also influenced by the finite dot thickness: the core size is shrunk close to both surfaces to minimize surface magnetic charges. The surface and mid-height core half-width are $2 \lambda_{\text {ex. }}$ and $3.4 \lambda_{\text {ex. }}$, respectively, similar to the values predicted for $60 \mathrm{~nm}$ thickness using a variational model and a perfectly soft material: $2.2 \lambda_{\text {ex }}$. and $5 \lambda_{\text {ex. }}$, respectively [1], with $\lambda_{\text {ex. }}=\sqrt{2 A / \mu_{0} M_{\mathrm{s}}^{2}}\left(\lambda_{\text {ex. }}=3.3 \mathrm{~nm}\right.$ for Fe). Note also that the mid-height core seems to stretch along $y$ and reproduce the short segment of wall predicted by the $\mathrm{VdB}$ model (Fig. 11 d). If that piece of $\mathrm{VdB}$ segment was longer it is probable that each vortex would lower its symmetry and break into a short Bloch wall, like in the LS.

Let us now comment on why two remanent states are observed in similar dots, despite the fact that the DS has a higher energy than the LS (Table I). This is related to a long standing issue $[1,13,14]$ : the demagnetized state of a system depends on its magnetic history. Here the LS has one single magnetic structure (a domain wall) whereas the DS has two structures (vortices). From this we expect that the selection of the LS or DS depends on the number of vortices that enter the dot during the demagnetization process. We indeed checked this by numerical simulation with the field applied $0.1^{\circ}$-in-plane-off [001]: the DS is selected for regular simulations (two vortices enter the dot at two symmetric nucleation points), whereas the LS can be selected by breaking the dot's symmetry, by means of a slightly asymmetric shape for example. In most experiments extrinsic parameters such as roughness and defects, and thermal activation, must influence the number and entry points of vortices. We expect 

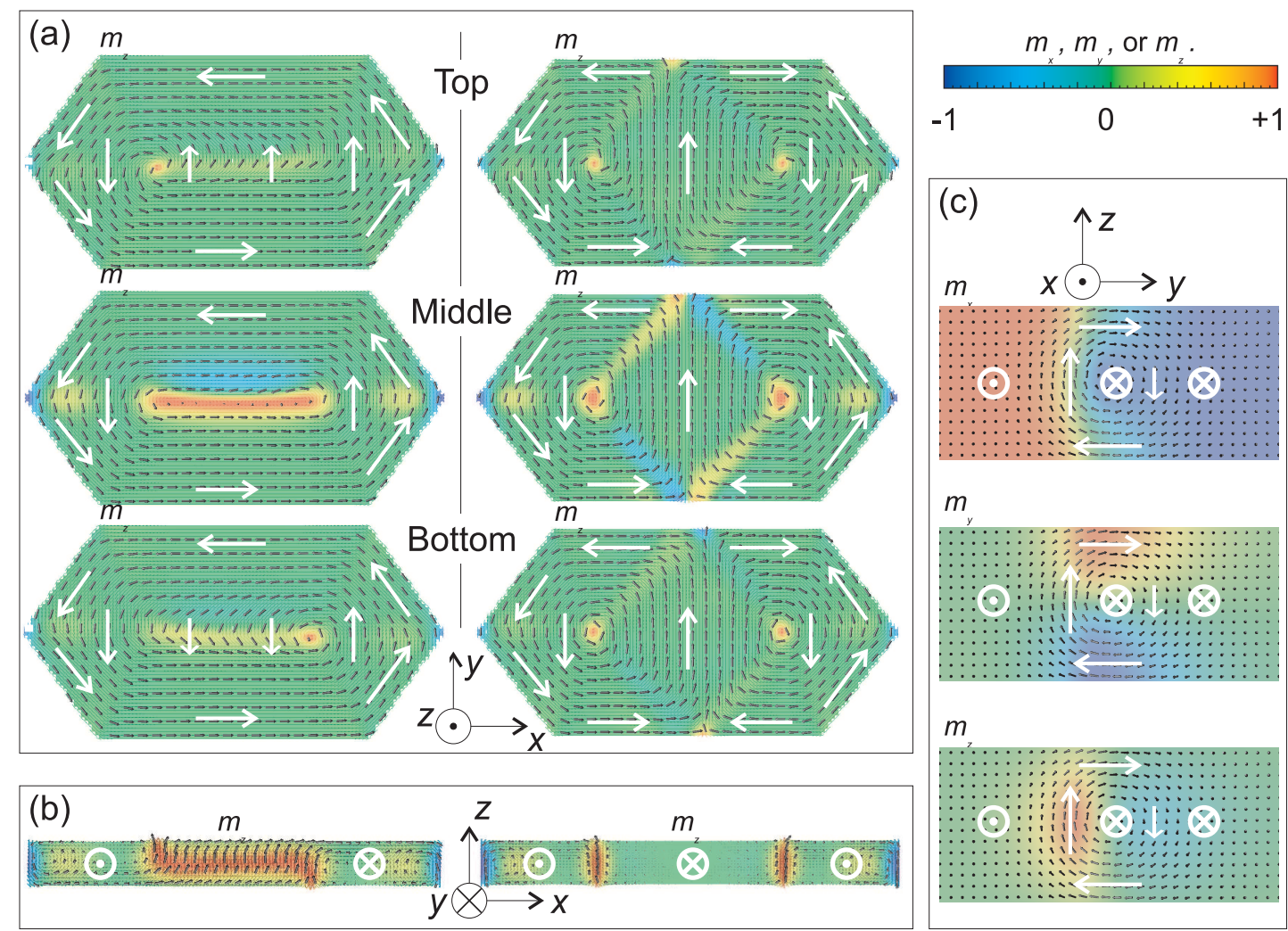

Fig. 2 - Simulated cross-sections of Landau (left in a-b) and diamond (right in a-b) states. $x=[001]$, $y=[1 \overline{1} 0], z=(110)$. White arrows sketch the in-plane magnetization direction. The color code applies to $m_{z}$ in (a-b) and to successively all directions in (c). (a) top, middle and bottom plane views (b) middle cross-sections in the $x z$ plane (c) a partial middle cross-section of size $60 \times 130 \mathrm{~nm}$ in the $y z$ plane.

that by using model dots as those presented here the nucleation is determined mostly by intrinsic parameters such as shape and external field history, making the comparison between experiments and simulations more relevant than for complex systems.

We finally discuss initial susceptibility values and magnetization energies, that are often used to estimate demagnetizing coefficients in large samples. We stress that some corrections must be taken into account for sub-micron size dots. Let us call $U$ the internal energy per unit volume of a system (including exchange $U_{\text {ex }}$, anisotropy $U_{\text {an }}$ and self-demagnetizing $U_{\mathrm{d}}$ energies) and $G=U-T S-\mu_{0} H_{0} m$ its free enthalpy. $H_{0}$ is a uniform external field and $m$ the average magnetization component along the field direction. At $0 \mathrm{~K}$ we have $\mathrm{d} U=\mu_{0} H_{0} \mathrm{~d} m$ and $\mathrm{d} G=-\mu_{0} m \mathrm{~d} H_{0}$. If equilibrium is achieved at each step of a magnetization process (no hysteresis) from zero field to a field high enough to nearly perfectly align all spins, that we call saturation field $H_{0}^{\text {sat }}$, then the area below the magnetization curve equals $\mu_{0} \int_{0}^{H_{0}^{\text {sat }}} m \mathrm{~d} H_{0}=$ $G(0)-G\left(H_{0}^{\text {sat }}\right)$ (Fig. 3). Note that $G(H)$ is continuous for non-hysteretic magnetization jumps, making the above integral valid for any non-hysteretic magnetization curve. From the definition of $G$ and given that $\int_{0}^{H_{0}^{\text {sat }}} m \mathrm{~d} H_{0}+\int_{0}^{M_{\mathrm{s}}} H_{0} \mathrm{~d} m=H_{0}^{\mathrm{sat}} M_{\mathrm{s}}$ it comes that the area 


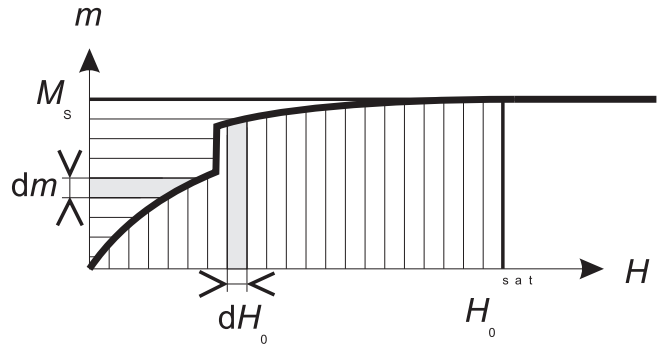

Fig. 3 - Illustration of $M_{\mathrm{s}}, H_{0}^{\text {sat }}, \int_{0}^{H_{0}^{\mathrm{sat}}} m \mathrm{~d} H_{0}$ (vertical hatches) and $\int_{0}^{M_{\mathrm{s}}} H_{0} \mathrm{~d} m$ (horizontal hatches).

above the magnetization curve is

$$
\mu_{0} \int_{0}^{M_{\mathrm{s}}} H_{0} \mathrm{~d} m=\frac{1}{2} \mu_{0} M_{\mathrm{s}}^{2} N_{i}+U_{\mathrm{an}}\left(H_{0}^{\mathrm{sat}}\right)-U(0) .
$$

We define the demagnetizing coefficient $N_{i}$ along the considered field direction as $U_{\mathrm{d}}\left(H_{0}^{\text {sat }}\right)=$ $-\frac{1}{2} \mu_{0} \mathbf{M}_{\mathrm{s}} \cdot \int \mathbf{H}_{\mathrm{d}} \frac{\mathrm{d} v}{V}=\frac{1}{2} \mu_{0} M_{\mathrm{s}}^{2} N_{i}$, where $\mathbf{M}_{\mathrm{s}}$ is uniform. This definition is valid not only for three-dimensional ellipsoids (and other shapes of degree less than 2), but for arbitrary volume shapes provided that uniform magnetization is assumed, even if $\mathbf{H}_{\mathrm{d}}$ is not uniform. With this definition $\Sigma N_{i}=1$ for any three perpendicular directions. Besides, Eq. (1) is general. It is not restricted to samples subdivided in fine domains like in the 'phase theory' ( [1], page 182 and included references). Eq. (11) is therefore suitable to describe flat dots with a small number of domains. When these dots are large exchange, anisotropy energy and hysteretic effects can be neglected, so that Eq. (1) boils down to $\mu_{0} \int_{0}^{M_{\mathrm{s}}} H_{0} \mathrm{~d} m=\frac{1}{2} \mu_{0} M_{\mathrm{s}}^{2} N_{i}$ and yields a straightforward determination of $N_{i}$ from hysteresis loops. However for sub-micron size dots the exchange and dipolar energy of the magnetic walls and vortices should no more be neglected, yielding a non-zero positive $U(0)$. Then, $\mu_{0} \int_{0}^{M_{\mathrm{s}}} H_{0} \mathrm{~d} m$ underestimates the true value of $N_{i}$. Besides, $U(0)$ is larger in dots of compact shape (all $N_{i}$ 's of similar magnitude) because dipolar energy is larger than in flat elements. Finally, in a dot with a given geometry, the most severe relative underestimation is expected when applying the field along an axis with small $N_{i}$, i.e. when the magnetizing energy is the weakest.

Our simulated and experimental results are gathered in Tables 盘. Although the dot's size is well above the exchange length (the length scale of magnetic vortices), calculation tells us (Tab.II, 1.6-7) that $U(0)$ amounts to $20 \%$ of $\frac{1}{2} \mu_{0} M_{\mathrm{s}}^{2} N_{\text {[001] }}$, the easiest shape magnetic axis. For calculations we checked on the DS along [001] that Eq. (11) is satisfied within $5 \%$ (Tables [1 1.1 ; II $1.3 ; 6)$. For experiments a discrepancy of around $10 \%$ is found. One reason for this slightly higher discrepancy may come from the continuous Fe ultrathin wetting layer arising during the so-called Stranski-Krastanov growth of Fe on Mo [11]. The magnetic contribution of this layer amounts to about $15 \%$ of the dots' signal. Besides, in experiments the evaluation of the non-hysteretic loop from hysteretic loops might suffer from systematic errors. In our case we estimated the non-hysteretic loop with the average of the magnetizing and demagnetizing curves, yielding a systematic error bar of $2 \%$.

The initial susceptibility $\chi_{i}$ is also sometimes used to determine the $N_{i}$ 's. In the case of large ellipsoids (and slabs, paraboloids ...) split into fine domains one can show that the magnetization process is strictly linear up to saturation, with $\chi_{i}=1 / N_{i}$. However this is no longer true if the surface of the system is not polynomial with a degree at most equal to 2 , or if the number of domains is small, because the demagnetizing field may not be uniform. 
TABLE I - The simulated total energy $U(0)$ and its origin for the DS and LS. For comparison, $\frac{1}{2} \mu_{0} M_{\mathrm{s}}^{2}=1.925 \times 10^{6} \mathrm{~J} / \mathrm{m}^{3}$.

\begin{tabular}{ccccc}
\hline Remanent state & $U(0)\left(10^{4} \mathrm{~J} / \mathrm{m}^{3}\right)$ & $U_{\mathrm{ex}}\left(10^{4} \mathrm{~J} / \mathrm{m}^{3}\right)$ & $U_{\mathrm{d}}\left(10^{4} \mathrm{~J} / \mathrm{m}^{3}\right)$ & $U_{\mathrm{an}}\left(10^{4} \mathrm{~J} / \mathrm{m}^{3}\right)$ \\
\hline Diamond (DS) & 3.381 & 1.488 & 0.959 & 0.934 \\
Landau (LS) & 2.810 & 1.415 & 0.707 & 0.688 \\
\hline
\end{tabular}

Both conditions are violated in flat dots, even of large lateral size. What happens in that case is that for sufficiently low external field the internal field $H_{0}+H_{\mathrm{d}}$ is zero $[3,15]$. It is straightforward to show that in this field expulsion regime the Zeeman energy equals $-2 U_{\mathrm{d}}$ and that $H_{0} \mathrm{~d} m=m \mathrm{~d} H_{0}$, which implies constant susceptibility. However above a critical external field a nonzero internal field progressively enters some regions of the sample [15], and the $m\left(H_{0}\right)$ curve becomes concave. Therefore, as the total area above the magnetization curve equals $\frac{1}{2} \mu_{0} M_{\mathrm{s}}^{2} N_{i}$, we have $1 / \chi<N_{i}$, and the difference depends on the exact sample shape. However experimental observation suggests that the disagreement is small and the linear regime extends close to $H_{0}^{\text {sat }}$. To our knowledge there is surprisingly no theoretical argument that can explain this observation. The situation becomes even more complex for micronsize dots as the non-hysteretic curve may experience a noticeable jump close to saturation, because magnetic walls and vortices are not expelled continuously from the dot. In that case it is not clear whether $1 / \chi$ should be smaller or greater than $N_{i}$. The discrepancy should increase for smaller dots. To settle ideas, let us refer the reader to a one parameter variational model that describes flat disks of soft magnetic material, that predicts that $\chi=$ $1 / N+O\left(R / \lambda_{\text {ex. }}\right)$, with $R$ being the disk radius [16]. In our simulations and experiments we find $1 / \chi<N_{i}$ for all directions. Finally, note that in macroscopic experiments the distribution of dots' aspect ratios may be important. Whereas distributions play no role in the magnetizing energy determination, assuming in a rough approach that $\chi$ scales with $1 / N$ one finds $\langle\chi\rangle=$ $\left(1+\sigma /\langle N\rangle^{2}\right) \chi(1 /\langle N\rangle)$ with $\sigma$ the aspect ratio variance. We expect a $10 \%$ systematic error in our case, $\sigma$ being deduced from AFM images.

To conclude we have used single-crystalline Fe dots self-assembled under UHV as a model system to test some micromagnetic aspects of sub-micron size magnetic dots. We showed that

TABLE II - Experimental and simulated figures for magnetizing energy $\mu_{0} \int H \mathrm{~d} m$, inverse susceptibility $1 / \chi$, shape factors $N_{i}$ and remanent energy $U(0)$. All figures are expressed in Tesla for comparison. This value can be viewed as the saturation field in the ideal case of strictly linear magnetization up to saturation. Multiplying with $M_{\mathrm{s}} / 2$ yields energies. The experimental figures are different from some previously released [11] owing to more precise magnetic measurements reported here.

\begin{tabular}{|l|c|rrr|rr|}
\hline & & \multicolumn{3}{|c|}{ Direction } & \multicolumn{2}{c|}{ Sum } \\
\cline { 3 - 6 } & & {$[001]$} & {$[1-10]$} & $(110)$ & Raw & Normalized to $2.2 \mathrm{~T}$ \\
\hline \multirow{2}{*}{ Experiments } & $2 \mu_{0} \int H \frac{\mathrm{d} m}{M_{\mathrm{s}}}$ & $0.206 \mathrm{~T}$ & $0.398 \mathrm{~T}$ & $1.28 \mathrm{~T}$ & $1.884 \mathrm{~T}$ & 0.86 \\
& $1 / \chi$ & $0.140 \mathrm{~T}$ & $0.325 \mathrm{~T}$ & $1.01 \mathrm{~T}$ & $1.49 \mathrm{~T}$ & 0.68 \\
\hline \multirow{5}{*}{ Calculations } & $2 \mu_{0} \int H \frac{\mathrm{d} m_{\mathrm{DS}}}{M_{\mathrm{s}}}$ & $0.166 \mathrm{~T}$ & not calc. & not calc. & not calc. & not calc. \\
& $1 / \chi_{\mathrm{DS}}$ & $0.130 \mathrm{~T}$ & $0.359 \mathrm{~T}$ & $1.407 \mathrm{~T}$ & $1.896 \mathrm{~T}$ & 0.861 \\
& $1 / \chi_{\mathrm{LS}}$ & $0.165 \mathrm{~T}$ & $0.346 \mathrm{~T}$ & $1.426 \mathrm{~T}$ & $1.937 \mathrm{~T}$ & 0.880 \\
& $N$ & $0.194 \mathrm{~T}$ & $0.386 \mathrm{~T}$ & $1.621 \mathrm{~T}$ & $2.2 \mathrm{~T}$ & 1 \\
& $U_{\mathrm{DS}}(0)$ & $0.039 \mathrm{~T}$ & $0.039 \mathrm{~T}$ & $0.039 \mathrm{~T}$ & irrelevant & irrelevant \\
\hline
\end{tabular}


the Van den Berg construction may remain perfectly relevant although all criteria for applicability are by far violated. We also argued, and showed experimentally and using simulations, that procedures for evaluating demagnetizing factors $N_{i}$ in bulk samples underestimate the $N_{i}$ 's in sub-micron size dots. A correction related to the energy of the remanent state must be included for the procedure based on loop-area determination, whereas the procedure based on the inverse initial susceptibility may lead to unpredictable systematic errors.

We acknowledge useful discussions of magnetization processes with J.-L. Porteseil (LLN).

We are grateful to P. David and V. Santonacci for their invaluable technical support and to N. Collins for the graphic visualization tools.

\section{REFERENCES}

[1] A. Hubert, R. Schäfer, Magnetic domains. The analysis of magnetic microstructures, Springer, Berlin, 1999.

[2] H. A. M. V. den Berg, J. Magn. Magn. Mater. 44 (1984) 207.

[3] P. Bryant, H. Suhl, Micromagnetic below saturation, J. Appl. Phys. 66 (1989) 9.

[4] J. Raabe, R. Pulwey, R. Sattler, T. Schweinböck, J. Zweck, D. Weiss, Magnetization pattern of ferromagnetic nanodisks, J. Appl. Phys. 88 (2000) 4437.

[5] T. Shinjo, T. Okuno, R. Hassdorf, K. Shigeto, T. Ono, Magnetic vortex core observation in circular dots of permalloy, Science 289 (2000) 930.

[6] R. P. Cowburn, M. E. Welland, Micromagnetics of the single-domain state of square ferromagnetic nanostructures, Phys. Rev. B 58 (14) (1998) 9217-9226.

[7] K. Y. Guslienko, K. L. Metlov, Evolution and stability of a magnetic vortex in a small cylindrical ferromagnetic particle under applied field, Phys. Rev. B 63 (2001) 100493(R).

[8] O. Fruchart, J.-C. Toussaint, B. Kevorkian, Micromagnetic model of non-collective magnetization reversal in ultrathin magnetic dots with in-plane uniaxial anisotropy, Phys. Rev. B 63 (2001) 174418.

[9] J. G. Deak, R. H. Koch, The effect of edge roughness on magnetization reversal in micron-sized permalloy thin films, J. Magn. Magn. Mater. 213 (2000) 25.

[10] R. P. Cowburn, Property variation with shape in magnetic nanoelements, J. Phys. D: Appl. Phys. 33 (2000) R1-R16.

[11] P. Jubert, O. Fruchart, C. Meyer, Self-assembled growth of facetted epitaxial fe(110) islands on mo(110), Phys. Rev. B 64 (2001) 115419.

[12] K. Ramstöck, T. Leibl, A. Hubert, J. Magn. Magn. Mater. 135 (1997) 97.

[13] W. Rave, A. Hubert, Magnetic ground state of a thin-film element, IEEE Trans. Magn. 36 (2001) 3886.

[14] J. Shi, S. Tehrani, M. R. Scheinfein, Geometry dependence of magnetization vortices in patterned submicron nife elements, Appl. Phys. Lett. 76 (18) (2000) 2588.

[15] A. DeSimone, R. V. Kohn, S. Müller, F. Otto, R. Schäfer, Two-dimensional modelling of soft ferromagnetic films, Proc. Roy. Soc. Lond. A 457 (2001) 2983-2991.

[16] K. Y. Guslienko, V. Novosad, Y. Otani, H. Shima, K. Fukamichi, Field evolution of magnetic vortex state in ferromagnetic disks, Appl. Phys. Lett. 78 (2001) 3848. 